\title{
Investigating the effect of thickness and frequency spacing on multi-frequency acoustic kinoforms
}

\author{
Michael D. Brown, Ben T. Cox and Bradley E. Treeby \\ Department of Medical Physics and Biomedical Engineering, University College London, London, UK \\ Email: michael.brown.13@ucl.ac.uk
}

\begin{abstract}
The generation of complex diffraction limited acoustic fields from a simple planar transducer is possible using cheap 3-D printable kinoforms. This approach is extremely promising for several areas of physical acoustics. However, one drawback is that the acoustic field generated from a given kinoform is fixed, limiting flexibility. In this work, multi-frequency acoustic kinoforms are investigated as a means to circumvent that limitation. These are kinoforms designed to generate different distributions of pressure at a target depth when driven at particular design frequencies. An optimisation approach based on direct search for the design of these structures from a set of input frequencies and target distributions is briefly described. The effect of different parameters of the kinoform on the performance are then established. These include the maximum thickness, frequency spacing and target depth. It is found that the maximum thickness has to be limited to avoid significant aberrations, the frequency spacing should be maximised within the usable bandwidth of the transducer, and the optimal thickness is influenced by the choice of target depth. The thin phase approximation is also shown to be increasingly inaccurate for increasing element thicknesses.
\end{abstract}

Index Terms-Holography, Kinoforms, Frequency multiplexing, 3-D printing

\section{INTRODUCTION}

In recent years, the manipulation of high frequency acoustic fields in 3-D has attracted increasing interest for applications in both physical acoustics and biomedical ultrasound [1, 2]. Recently, a new approach for the generation of complex diffraction limited acoustic fields from a planar transducer was introduced [2]. This uses 3-D printed acoustic kinoforms, structures with a spatially varying thickness that can be attached to the front of planar transducers. The difference in sound speed between the 3-D printed material $c_{h}$ and the surrounding medium $c_{c}$ results in a spatially varying phase offset dependent on the thickness of the structure at each point. This enables the continuous wave output of a planar single element transducer to be diffracted into the desired distribution of acoustic pressure.

Acoustic kinoforms are cheap to fabricate, require just a single transducer, and scale easily up to several $\mathrm{MHz}$ with the resolution of contemporary 3-D printers. However, one drawback is the fixed nature of the acoustic field generated by each kinoform, which limits flexibility. A way to overcome this is to adapt a technique from optical holography [3] and encode several target acoustic distributions onto the same kinoform for

The authors would like to thank the Engineering and Physical Sciences Research Council (EPSRC) for funding the work. different driving frequencies. The field can then be changed by varying the driving frequency of the transducer. The goal of this work is to introduce an approach for designing these multifrequency kinoforms and to analyse the effect of different parameters on their performance.

\section{MUlTi-FREQUENCY KINOFORMS}

Consider a kinoform with a spatially varying thickness described by $h(x, y)$ attached to the front face of a planar transducer lying in the $z=0$ plane. When driven at a single frequency $f$, the phase offset introduced by the kinoform can be described by

$$
\Delta \phi(x, y)=2 \pi f\left(\frac{1}{c_{c}}-\frac{1}{c_{h}}\right)\left(h_{m}-h(x, y)\right)
$$

where $h_{m}$ is the maximum thickness of the kinoform. This approximates the kinoform as an infinitely thin phase screen, neglecting any absorption and scattering effects.

The task when designing a multi-frequency kinoform is to find a spatially varying surface profile $h(x, y)$ that maps a set of design frequencies $f_{n}$ onto different phase distributions $\Delta \phi_{n}(x, y)$ that diffract the plane wave into different target pressure distributions $p_{n}\left(x, y, z_{d}\right)$. Here $z_{d}$ is the depth of the target plane. This concept is illustrated in Fig. 1.

A variety of algorithms have been developed that address the equivalent problem in optics. Two simple approaches are to divide the aperture into separate regions for each frequency or to exploit the chromatic variation in focal length of Fresnel kinoforms that occurs due to the wavelength dependence of the phase offsets from free-space propagation [3, 4]. Other works have used optimisation methods that search for a thickness at each position by maximising a quality metric based on the target field for each frequency. Several optimisation approaches have been used including direct search [5] and the optimal-rotation-angle (ORA) method [4].

For this work, the direct search approach outlined by Kim et al [5] was modified based on a previous study on the design of surface profiles for the generation of patterned optoacoustic fields [6]. Rather than define target pressure distributions $p_{n}\left(x, y, z_{d}\right)$, sets of target points $p_{n}=\left\{\left(x_{m}, y_{m}, z_{d}\right), m=\right.$ $1, \ldots, M\}_{n}$ were defined for each frequency, with the aim of maximising the amplitude at these points. The kinoform coordinates and height levels were discretised using a spacing $\Delta$. For modelling the field, the Rayleigh-Sommerfeld integral was used in conjunction with Eq. 1 to calculate the complex 


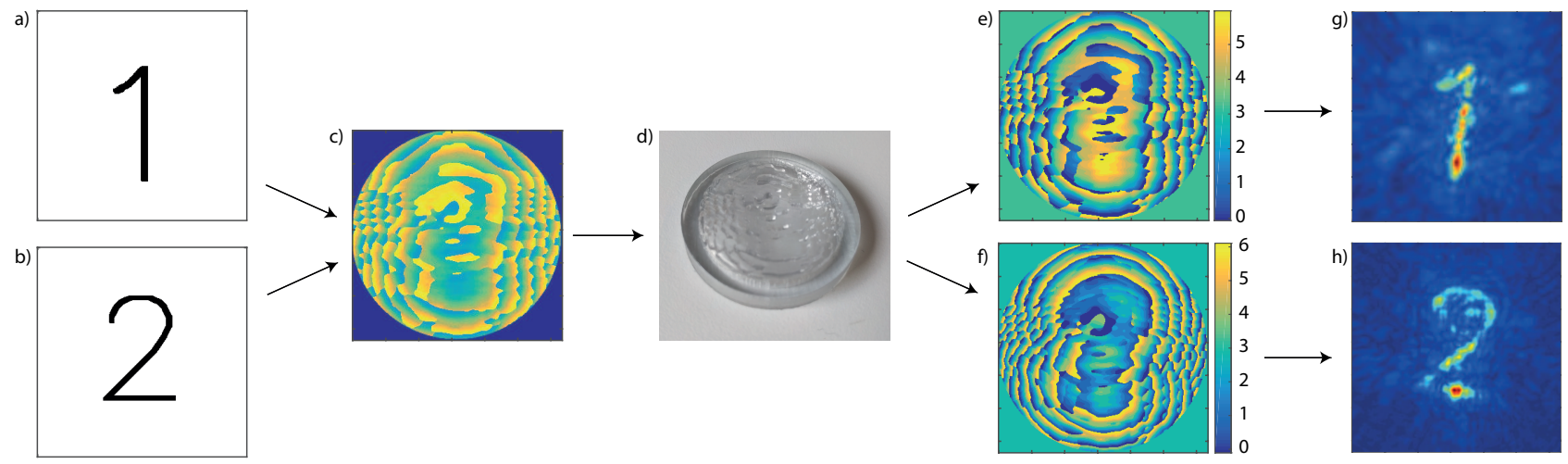

Fig. 1. Illustrative figure demonstrating the concept of multi-frequency kinoforms. (a-b) Target distributions are defined for each driving frequency. (c) A direct search approach is used to calculate the kinoform. (d) Kinoform is fabricated via 3-D printing. (e-f) Kinoform is attached to a transducer where it generates different phase offsets for each frequency. (g) Output field is characterised and compared to the target distributions.

pressure at the target points $p_{n}$ for each frequency. The optimisation was initialised with a flat surface and proceeded by perturbing the height of individual pixels to search for a kinoform profile which minimised a cost function. In this work, a cost function that rewarded increases in the average pressure across the target points was used. The output of the optimisation is a 2-D matrix $h_{i j}$ that describes the variation in height across the kinoform surface.

A kinoform designed using this algorithm is shown in Fig. 1. This was designed for a $3.1 \mathrm{~cm}$ PZT piston transducer (Olympus, Japan) with a centre frequency of $2.25 \mathrm{MHz}$. The target patterns, which consisted of the numerals ' 1 ' and ' 2 ', can be seen in Fig. 1(a-b). The patterns were encoded onto the frequencies 1.9 and $3.1 \mathrm{MHz}$. The image depth $z_{d}$ was $2.5 \mathrm{~cm}$. The kinoform height and coordinates were discretised using a spacing $\Delta$ of $100 \mu \mathrm{m}$ and the maximum thickness $h_{m}$ was set to $2 \mathrm{~mm}$. The kinoform was printed using a highresolution polyjet printer (Objet350 Connex, Stratasys, Eden Prairie, MN, USA) using veroClear as the substrate.

\section{OPTIMISATION PARAMETERS}

\section{A. Crosstalk}

The efficiency with which multiple target acoustic distributions can be created by a multi-frequency kinoform is highly dependent on a number of design parameters. For example, it can be seen from Eq. 1 that the phase offset generated for each frequency is identical aside from a scaling factor. For low maximum modulation depths (on the order of $2 \pi$ ) and narrowly spaced frequencies, this means the phase offsets generated for different design frequencies vary only slightly. As a result, each frequency has a similar diffraction pattern and each distribution encoded on the kinoform is generated in the field of each design frequency. This is referred to as crosstalk.

Fortunately, near-field kinoforms have a chromatic variation in focal depth that occurs due to the wavelength dependence of the phase offsets generated by free-space propagation [7]. This means that while all encoded patterns will appear in the field simultaneously, the patterns for other design frequencies will be out-of-plane. For a target depth $z_{d}$, the depth $z_{c}$ at which each pattern is realised can be approximated by the ratio of the design and driving frequencies $z_{c}=z_{d} f_{d r} / f_{d e}$, where $f_{d e}$ is the design frequency for the pattern and $f_{d r}$ is the frequency driving the transducer. This property is integral to the operation of the multi-frequency kinoforms in this work.

The amplitude of the crosstalk can in principle be reduced by observing that the diffracted field is determined by the $2 \pi$ modulus of the phase rather than the absolute value. Therefore, increasing the thickness and using a kinoform with a high maximum phase modulation depth $>4 \pi$ can allow more distinct phase patterns to be generated for each frequency, as a given value of $\phi_{1}$ will correspond to multiple values of $\phi_{2}$ [4]. A numerical investigation was carried out to establish the effect of the maximum thickness $h_{m}$, the separation of the encoded frequencies $f_{n}$, and the image depth $z_{d}$, on the performance of the hologram.

\section{B. Numerical experiment design}

All experiments were carried out for a kinoform with a diameter of $3.1 \mathrm{~cm}$ discretised with a spacing $\Delta$ of $100 \mu \mathrm{m}$. Two binary patterns were encoded in each case: the numerals ' 1 ' and ' 2 ' seen in Fig. 1(a-b). The sound speed and density were set to $2495 \mathrm{~ms}^{-1}$ and $1190 \mathrm{kgm}^{-3}$.

Two approaches were used to calculate the field from each kinoform. For the first method, the kinoforms were approximated as infinitely thin phase elements that imparted phase offsets given by Eq. 1. A uniform incident pressure was assumed and the angular spectrum approach (ASA) was then used to propagate the field to the target depth [8]. However, for kinoforms possessing narrow fringe spacing (high spatial frequencies) and substantial fringe heights, the thin element approximation can break down. This introduces aberrations to the field compared to the ideal assumption [9]. To assess the severity of these effects, for the second method propagation through each kinoform was also simulated using a full wave model, the k-Wave toolbox [10]. This accounted for medium heterogeneities and the physical structure of the kinoform.

Two key metrics were of interest. The signal to noise ratio (SNR) achieved in the target plane for each encoded 
pattern and the crosstalk between the patterns. To calculate the metrics, the images of ' 1 ' and ' 2 ' were used to define a foreground and a background for both patterns. The SNR was defined as the ratio of the average pressure across the foreground and the background. This was evaluated at the target depth for both models and each design frequency. The crosstalk was defined using the same method, except the SNR of the ' 2 ' was evaluated in the field of the ' 1 ' and viceversa. This was measured for only the full-wave model. As the crosstalk image depth varies from the target depth, the metric was evaluated for all depths and the plane with the highest value was selected for each frequency. For each test, the SNR and crosstalk were averaged across both patterns to give a single value.

\section{Maximum thickness and modulation depth}

As discussed earlier, the maximum thickness $h_{m}$ or the maximum phase modulation depth $\Delta \phi$ has a significant impact on the resulting field. If the different frequencies can be considered to have ideal phase distributions $\left(\Phi_{1}, \ldots, \Phi_{n}\right)$ then these can be approximated with much lower error for high modulation depths [11]. To test the effect of $h_{m}$, a set of kinoforms were calculated in which $h_{m}$ was varied between 1-6 mm. In each case, the two design frequencies were set to 2 and $3 \mathrm{MHz}$ and the image depth was set to $2.5 \mathrm{~cm}$.

Figure 2(a) shows the variation in SNR and crosstalk as a function of maximum thickness. As expected due to the reduction in phase error, the SNR for the ASA model improves with increasing $h_{m}$ and the full-wave crosstalk decreases. Both converge for $h_{m}>4 \mathrm{~mm}$ or a $4 \pi$ modulation for $2 \mathrm{MHz}$, suggesting the optimisation can find few further improvements. However, the full-wave model SNR behaves very differently. For $h_{m}$ between 1 and $\sim 1.8 \mathrm{~mm}$ it increases. This is followed by a slow drop between $1.8-4 \mathrm{~mm}$, then a rapid falloff between 4-6 mm. This occurs because the thin element approximation used for the ASA model becomes increasingly inaccurate with increasing $h_{m}$, as the thickness increases and the kinoform structures have higher spatial frequencies. After $4 \mathrm{~mm}$, extremely rapid jumps appear in the kinoform height causing a complete breakdown in the optimisation forward model. This can be seen in Fig. 2(c). These results suggest that it is necessary to limit $h_{m}$ to ensure the optimisation converges to usable kinoforms.

\section{Frequency spacing}

The spacing of the design frequencies is also an important parameter. In practice, it is constrained by the transducer bandwidth, however, the choice of frequencies within that range can have a significant impact on performance. To investigate, kinoforms were calculated with the design frequency for the ' 1 ' fixed at $1.5 \mathrm{MHz}$, and the design frequency for the ' 2 ' increased in increments of $100 \mathrm{kHz}$ starting at $1.6 \mathrm{MHz}$. This was done for $h_{m}$ set to 2.5 or $5 \mathrm{~mm}$, corresponding to modulation depths of $2 \pi$ and $4 \pi$ at $1.5 \mathrm{MHz}$.

Figure 3(a) shows the variation in SNR and crosstalk for a maximum thickness of $2.5 \mathrm{~mm}$. This shows that as the ratio
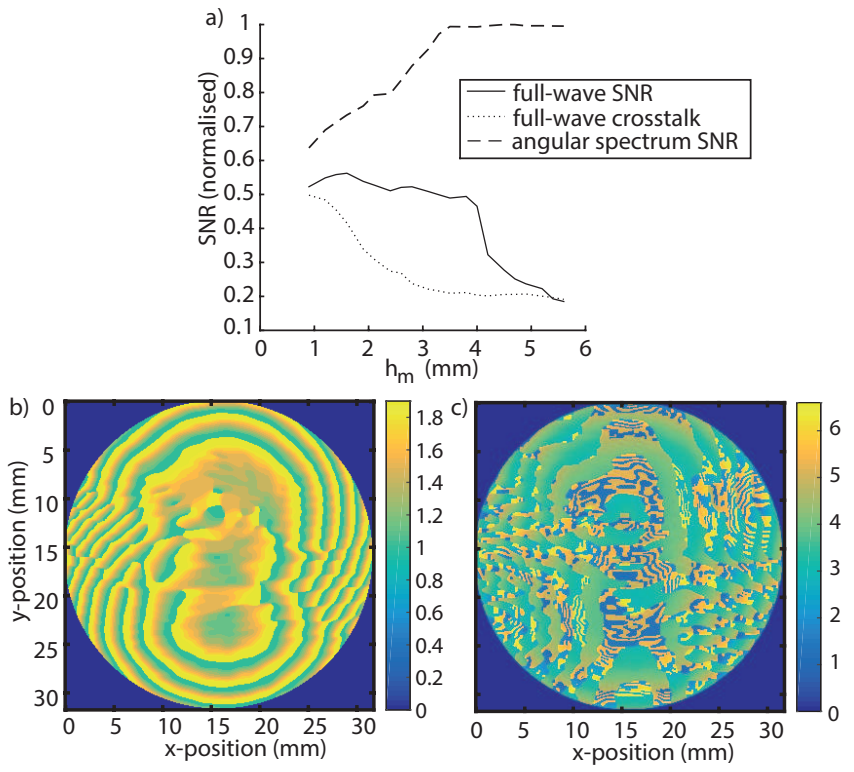

Fig. 2. (a) Variation in average SNR and crosstalk for the two pattern kinoform as a function of maximum thickness $h_{m}$ normalised to the peak value. (b) Kinoform calculated for $h_{m}$ of $1 \mathrm{~mm}$. (c) Kinoform calculated for $h_{m}$ of $6 \mathrm{~mm}$. The rapid jumps in height as the modulation depth increases are apparent.
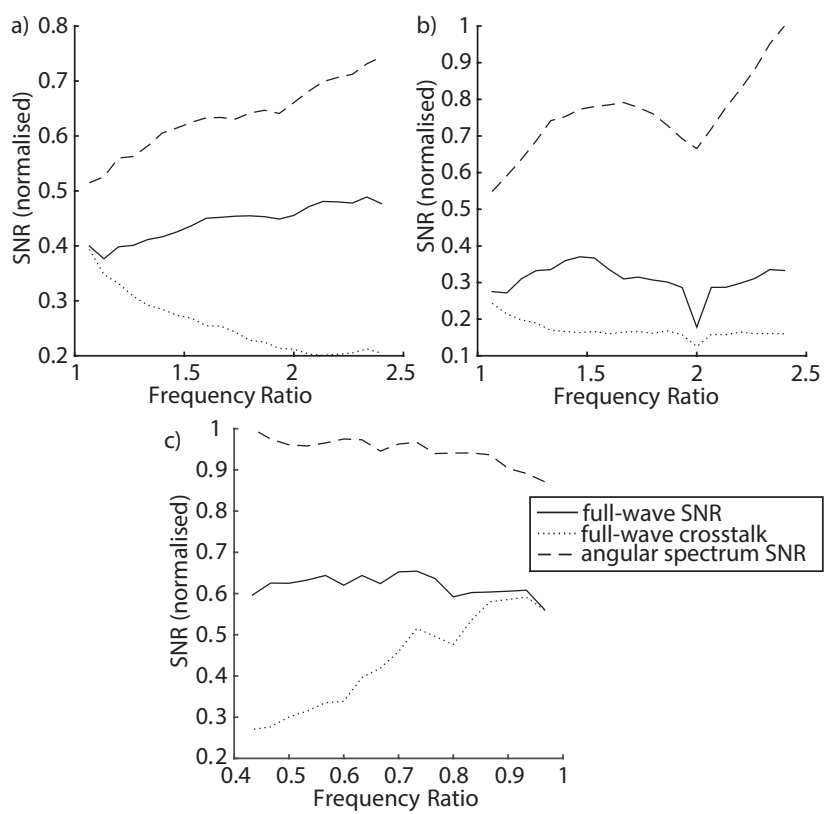

Fig. 3. Variation in average SNR and crosstalk for the two pattern kinoform as a function of the frequency ratio. (a) Variation for $h_{m} 2.5 \mathrm{~cm}$ for a constrained lower frequency, normalised to peak across (a) and (b). (b) Variation for $h_{m}$ $5 \mathrm{~cm}$ for a constrained lower frequency, normalised to peak across (a) and (b). (c) Variation for constrained upper frequency normalised to peak in (c).

of frequencies increases, the overall SNR increases and the crosstalk decreases for both the full-wave and ASA model. This is because the phase modulation depth increases for the higher frequency (due to its shorter wavelength) reducing the average phase error. The higher frequency also has a smaller point spread function (PSF) improving the fidelity of the target pattern. The decrease in the crosstalk is again due to 
less similarity between the phase distributions as wavelength difference and modulation depth increase.

Figure 3(b) shows the variation for a maximum thickness of $5 \mathrm{~mm}$, which is notably different. Initially, as the ratio of frequencies increases from 1 to $\sim 1.6$, both the ASA and full-wave SNR increase. However, as the higher frequency approaches the second harmonic, both start to decrease. This decrease is due to how the phase offsets for each frequency are distributed relative to each other and can be explained by considering two cases: $f_{2}=2 f_{1}$ and $f_{2}=1.5 f_{1}$. For $f_{2}=2 f_{1}$, for a given phase value $\phi_{2}$ there are only 2 possible phase values for $\phi_{1}$, despite the $4 \pi$ modulation depth. For $f_{2}=1.5 f_{1}$, there are 3 possible $\phi_{1}$ values for each $\phi_{2}$. Thus despite the lower modulation depth, the latter will have a lower average phase error [12]. These results suggest that for low modulation depths and fixed lower frequency the spacing should be maximised, while for higher modulation depths harmonics should be avoided.

Next, the case of fixed upper frequency was investigated. To test this, the frequency of the ' 2 ' was fixed at $3 \mathrm{MHz}$ and the frequency of the ' 1 ' was decreased in increments of $100 \mathrm{kHz}$ starting at $2.9 \mathrm{MHz}$. The maximum thickness $h_{m}$ was varied for each kinoform so that a $2 \pi$ modulation depth was provided for the ' 1 ' in each case. The results are shown in Fig. 3(c). In this case, the SNR for the ASA model still increases with increasing spacing, however, this happens more slowly. The initial improvement is principally due to the removal of in-plane crosstalk. It can be seen from Eq. (2) that for close spacing, the patterns overlap at the target depth. The subsequent improvement is again principally due to increases in the modulation depth. The improvements are offset by increases in the PSF for the lower frequency which decrease the fidelity of the ' 1 '. Slightly different results can be seen for the full-wave SNR, where a small initial increase is followed by stability. The SNR remains stable rather than increasing, as the inaccuracy of the ASA model increases due to the greater element thickness. This is partially offset by decreases in the kinoform spatial frequency.

\section{E. Target depth}

The variation in SNR with target depth $z_{d}$ is also interesting. Intuitively, the solution quality would largely be expected to increase as the target depth is reduced due to the larger numerical aperture. However, the fringe spacing (or spatial frequency) also increases simultaneously, increasing the abberations. To test the effect of image depth, kinoforms were calculated varying $z_{d}$ between $2-5 \mathrm{~cm}$ in increments of $3 \mathrm{~mm}$. This was done for $h_{m}$ of 2 and $3 \mathrm{~mm}$ and fixed design frequencies of 2 and $3 \mathrm{MHz}$.

Figure 4 shows the resulting variation. As expected, in both cases the ASA model SNR decreases with increasing target depth, due to the increasingly broad PSF. However, the fullwave model SNR increases up to a target depth of $4 \mathrm{~cm}$, as the reduction in aberrations compensates for the decrease in the fidelity of the target patterns. Similar trends are seen for both values of $h_{m}$ tested. In this case, the overall peak in
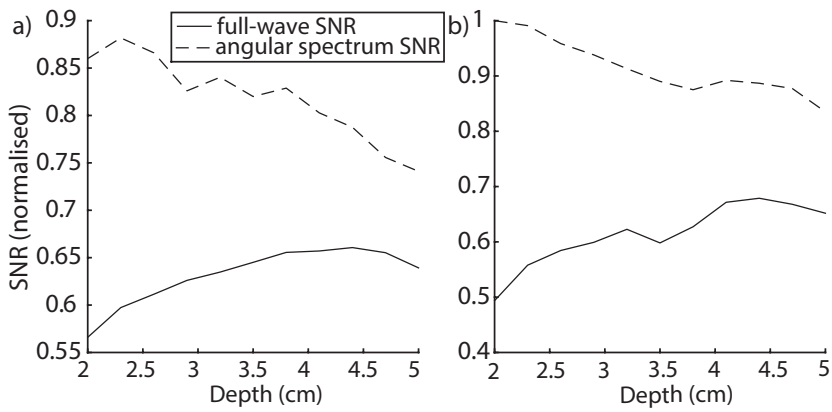

Fig. 4. Variation in average SNR for the two pattern kinoform as a function of the target depth for (a) $h_{m}$ of $2 \mathrm{~cm}$ (b) $h_{m}$ of $3 \mathrm{~cm}$. All lines normalised to peak across (a) and (b).

SNR occurs for a $h_{m}$ of $3 \mathrm{~mm}$ suggesting that the optimal modulation depth will vary for different problems.

\section{CONCLUSION}

This work has shown that multi-frequency acoustic kinoforms can be used to create at least two arbitrary acoustic distributions at a single target depth from a planar transducer. The effect of the maximum thickness, frequency spacing, and target depth on the resulting field has been established. It has also been shown that using a thin phase approximation to design multi-frequency kinoforms becomes increasingly inaccurate as the maximum thickness and fringe spacing increase. This work could have applications for several areas of physical acoustics in which targeted delivery of ultrasound is important, including particle manipulation and imaging.

\section{REFERENCES}

[1] Y. Hertzberg, O. Naor, A. Volovick, and S. Shoham, "Towards multifocal ultrasonic neural stimulation: Pattern generation algorithms", J. Neural Eng. 7, 056002 (2010).

[2] K. Melde, A. G. Mark, T. Qiu, and P. Fischerr, "Holograms for acoustics", Nature, 537, 518-522 (2016).

[3] M. Makowski, I. Ducin, K. Kakarenko, J. Suszek, M. Sypek, and A. Kolodziejczyk, "Simple holographic projection in color", Optics Express, 20, 25130-25136 (2012).

[4] J. Bengtsson, "Kinoforms designed to produce different fan-out patterns for two wavelengths", Appl. Opt., 37, 20112020 (1998).

[5] G. Kim, J. A. Domnguez-Caballero, and R. Menon, "Design and analysis of multi-wavelength diffractive optics," Opt. Express, 20, 28142823 (2012).

[6] M. D. Brown, D. I. Nikitichev, B. E. Treeby, B. T. Cox, "Generating arbitrary ultrasound fields with tailored optoacoustic surface profiles", Appl. Phys. Lett., 110, 094102 (2017).

[7] M. S. Millan, J. Oton, and E. Perez-Cabre, "Chromatic compensation of programmable Fresnel lenses", Optics Express, 14, 6226-6242 (2006).

[8] G. T. Clement and K Hynynen,"Field Characterization of therapeutic ultrasound phased arrays through forward and backward planar projection", J. Acoust. Soc. Am., 108(1), 441-446 (2000).

[9] D. A. Pommet, M. G. Moharam, and E. B. Grann, "Limits of scalar diffraction theory for diffractive phase elements", Opt. Soc. Am. A, 11, 1827-1834 (1993).

[10] B. E. Treeby and B. T. Cox, "k-Wave: MATLAB toolbox for the simulation and reconstruction of photoacoustic wave-fields", J. Biomed. Opt., 15, 021314 (2010).

[11] A. Jesacher, S. Bernet, and M. Ritsch-Marte, "Colored point spread function engineering for parallel confocal microscopy", Optics Express, 24, 27395-27402 (2016).

[12] A. J. Caley and M. R. Taghizadeh, "Analysis of the effects of bias phase and wavelength choice on the design of dual-wavelength diffractive optical elements", J. Opt. Soc. Am. A, 23, 193-198 (2006). 